\title{
Using Goal-Question-Metric (GQM) Approach to Assess Security in Cloud Storage
}

\author{
Fara Yahya ${ }^{(\bowtie)}$, Robert J. Walters, and Gary B. Wills \\ University of Southampton, Southampton, UK \\ fara.yahya@soton.ac.uk
}

\begin{abstract}
Assessing the security of data stored in cloud storage can be carried out by developing goal-based measurement items. The measurement items can be utilized to construct a security assessment model based on practical needs. The measurement items can assist in acquiring support decision making on the implementation of a security frameworks. This paper discusses the GoalQuestion-Metrics (GQM) approach and its application towards constructing measurement items for a security metric. It also attempts to provide practical guidance and example of measurements using GQM. An application of the GQM paradigm towards the development of a security metric is presented. The metrics obtained will assist organizations to meet their requirements for a cloud storage security framework.
\end{abstract}

Keywords: Cloud storage - Goal-Question-Metric · Security measurement · Security metric

\section{Introduction}

As data stored in the cloud become more complex, connected, and dynamic in nature, the related security management becomes more challenging. There is a need for systematic techniques with which to obtain quantitative evidence of the systems' security performance. Measurement is widely practiced in engineering and management as means of increasing the understanding of complex real world phenomena and enabling informed and adaptive decision-making. At the moment, domain-specific expertise is the standard knowledge used to manage security. There is a need for more general methods to provide measures for security management. Research on measuring security as a systematic discipline is still in its infancy, although there are examples of industrial security measurement practices. Security Metrics (SM) can be applied to provide security evidence for security engineering, risk and security management, and internal and external evaluation.

The main contributions of this paper is to develop a security assessment model that provides quantitative high level security assessments of cloud storage for IT security practitioners. The SM are expected to assess the relative level of security offered by organizations, cloud storage providers (CSPs) or cloud architectures.

The purpose of our paper is to present a Goal-Question-Metric approach that will support the definition of tailored SM to assess the security of data held in cloud storage. We will start by presenting how security is measured in computer science and cloud 
storage. Then, we will discuss cloud storage security challenges and security goals. Later, we will introduce GQM approach to derive SM related to the cloud storage context.

\section{Background}

Security is a key concern in society. The term is usually defined as a set of different concepts related to "protection" (Colobran 2016). Computer security means to protect information. It manages prevention and detection of unauthorized actions by users of a computer. Security overall covers mainly three aspects: confidentiality, integrity and availability (CIA). These aspects are the topmost considerations in designing a security measure. Currently, computer security has been extended to include security attributes such as authenticity, authorization, identification, and non-repudiation (Microsoft 2015). Each of these aspects refers to security objectives while security threats are specific to the systems (Gonzales et al. 2015). Despite the fact that security is key in society, several questions are still debatable (Baldwin 1997). How much security is needed? How could security be measured? Which security degree could be achieved? The term SM has a range of meanings, with no widely accepted definition. Measuring security is an interesting issue because the concept is challenging. SM reflect some security attributes quantitatively (Pendleton et al. 2016).

\section{Measuring Security in Cloud Storage}

Measuring security is important in order to predict future situations and take appropriate countermeasures in advance (Colobran 2016). A measurement is defined as " $a$ quantitatively expressed reduction of uncertainty based on one or more observations" (Hubbard 2014). Traditionally, security in computer science is based on objective indicators that supply a level of security. Subjective measures are used in fields of computer visualizations (Torkhani et al. 2015). They make sense when data is difficult to obtain; when the associated concepts are difficult to measure, e.g. "measuring user satisfaction etc." (Jahedi and Méndez 2014). Subjective measures can be collected from survey questions or by some sort of assessment made by experts. In the IT field there are also several subjective measures like the "usability" concept for example. Indeed measurements such as the "degree of understanding of security issues among computer users, remain somewhat subjective" (Payne 2006).

According to Cisco Global Cloud Index, cloud storage users will store 1.6 Gigabytes data per month by 2019, compared to 992 megabytes data per month in 2014 (Cisco 2014). The projection of cloud storage growth per user is presented in Fig. 1. With this trend, it has been shown that more and more data will reside in cloud storage and it is expected to grow further. As the cloud becomes the tool of choice for data storage services, the number of cloud storage providers (CSPs) is also increasing. From these providers, users have a wide selection of services available to move their data into the cloud. However, the responsibility for maintaining the security of sensitive data stored therein remains paramount (Zissis and Lekkas 2012). 


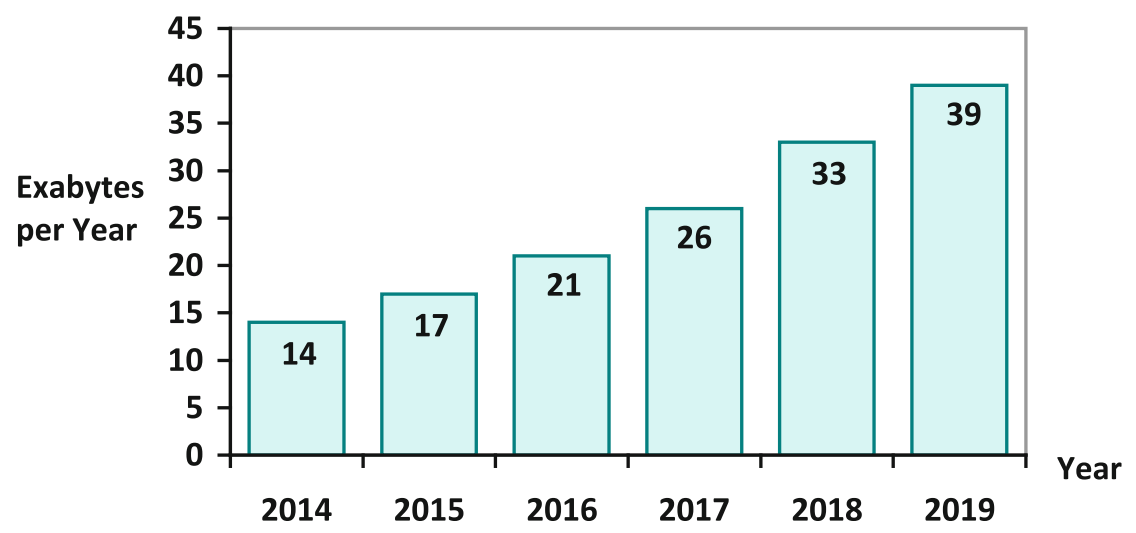

Fig. 1. The cloud storage growth per user (Cisco 2014)

Cloud storage provides a facility for those users who mainly require highly scalable storage on demand that is accessible globally. Despite the benefits cloud storage may bring to users through convenience and lower cost, it also may bring security concerns. These worries have been identified by various researchers (CSA 2010, 2013a; GTISC and GTRI 2013; Sabahi 2011; Shaikh and Haider 2011) who conclude that security concerns are increasing significantly every year.

Recent reports by Cloud Security Alliance (CSA 2013a) and Georgia Technology Information Security Center (GTISC and GTRI 2013) reveal a trend of insecure APIs, data loss and leakage concerns, as outsiders gain access to unencrypted data. Several simple methods are employed: a user password may be discovered by brute force, and unencrypted local files or folders located in the cloud can easily be accessed. The cloud service itself can also be compromised. In these cases, users and CSPs should implement security measures before the data is stored online.

CSPs have been implementing controls to secure access to sensitive data in the cloud, such as two-factor authentication, encryption, etc., making access to the data more difficult for attackers (Mather et al. 2009). Then again, an increase in security controls affects the usability of the data and therefore may cause the system to be avoided by users (Honan 2012; Zarandioon et al. 2012; Zhang and Chen 2012, Zhao and Yue 2014; Zissis and Lekkas 2012). Most CSPs are unwilling to reduce the efficiency of access to cloud storage because users expect equally efficient access into secured data as into plain text data. Security protection based on security components and mitigating its concerns is foreseen as one of the efforts to overcome this issue.

\section{Security Challenges in Cloud Storage}

According to an IDC IT Cloud survey, 74\% of IT executives and CIOs mentioned security as the top concern in cloud services. The cloud providers should be able to provide suitable security controls (Subashini and Kavitha 2011). Data security is an important factor for cloud adoption. An approach that provides real time data protection and blocks threats and isolate affected data in a Data Center is one of the ways to overcome data security issues (Chang and Ramachandran 2016). 
In the cloud, application software and databases are operated in large data centres. This poses several new security challenges (Singh et al. 2012; Wang et al. 2012). These challenges include but are not limited to the following (Ryan 2013; Srinivasan and Rodrigues 2012; Subashini and Kavitha 2011; Wang et al. 2012):

- accessibility vulnerabilities

- virtualization vulnerabilities

- web application and APIs vulnerabilities such as:

- SQL (Structured Query Language) injection

- cross-site scripting

- physical access issues

- privacy and control concerns as third parties have physical control of data

- identity and credential management issues

- issues associated with integrity and confidentiality such as data verification, tampering, data loss and theft

- issues related to authentication of devices and IP spoofing

Computer storage is where data is held. Storage has been divided into primary storage, which holds data in memory (RAM) and other "built in" devices such as the processor's L1 cache, and secondary storage, which holds data on hard disks, tapes, and other devices requiring input/output operations (Rouse 2005). Primary storage is much faster to access than secondary storage because of its proximity of the storage to the processor or because of the nature of the storage devices. On the other hand, secondary storage can hold much more data than primary storage, and this includes external hard disks and USB flash drives (Rouse 2005).

Cloud storage is known as utility storage if delivered through public cloud service providers (Wu et al. 2010). On the other hand, private service providers offer the same scalability, flexibility, and storage mechanism with restrictions or non-public access. Cloud storage runs on a virtualization platform providing end users and applications with a scalable and provisioned virtual storage architecture. Generally, cloud storage is accessed through an API (Ju et al. 2011; Wu et al. 2010).

\section{Definition}

Cloud storage is defined as a cloud computing model that stores data on distributed servers and is accessible anywhere through the Internet. A cloud service provider maintains, operates, and manages storage servers that are built using virtualization techniques (Wu et al. 2010).

\section{Architecture}

Cloud storage architectures contain a front end that exports an API to communicate with the backend storage ( $\mathrm{Ju}$ et al. 2011), as shown in Fig. 2. At this layer, there are Web service, file-based Internet SCSI or iSCSI front ends (Jones 2010). This layer is the first communication point between the user and the service provider. Users access 


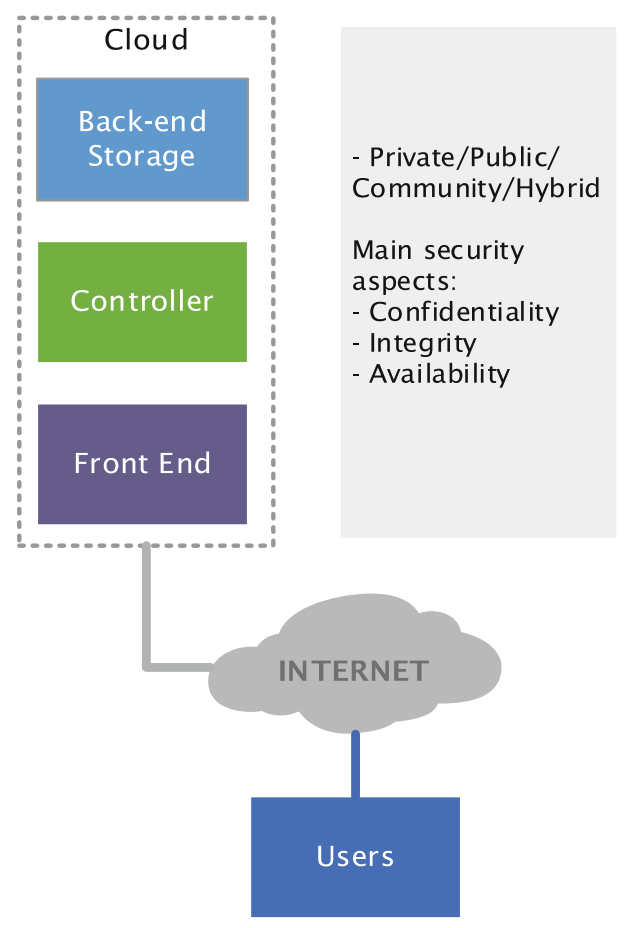

Fig. 2. Generic cloud storage architecture

the services using their credentials. The midpoint component is a layer called controller that interconnects the front end API to the backend storage. This layer has a variety of features such as virtualization, replication and data-placement algorithms with geographical location. Finally, the back-end consists of physical storage for data. This may be a central protocol that runs dedicated programs or a traditional back-end to the physical disks.

\section{Frameworks for Security}

A framework is a basic structure underlying a system or concept. It is a broad overview, outline, or skeleton of interlinked items which supports a particular approach to a specific objective and serves as a guide that can be modified as required by adding or deleting items (Firesmith 2004). IT security frameworks and standards can be helpful in addressing many areas such as encryption, application security, and disaster recovery. An information security framework is a series of documented processes that are used to define policies and procedures around the implementation and ongoing management of information security controls in an enterprise environment (Granneman 2013).

These frameworks are basically a blueprint for building an information security programme to manage risk and reduce vulnerabilities. Information security can utilise these frameworks to define and prioritise the tasks required to build security into an 
organization. Frameworks can also be customised to solve specific security problems to meet the required specifications and use (Firesmith 2004; Granneman 2013).

\section{Cloud Security Frameworks}

Several frameworks have addressed cloud security in general (Brock and Goscinski 2010; Firesmith 2004; Takabi et al. 2010; Zissis and Lekkas 2012), and cloud storage in particular (Mapp et al. 2014). Researchers have also applied security components as specific objectives. A review of existing frameworks for cloud security to obtain common security components is summarized in Table 1. We have also addressed issues and threats in cloud storage with security components (Yahya et al. 2014, 2015). Descriptions are presented below.

Table 1. Summary of security components from existing studies

\begin{tabular}{l|l|l|l|l|l}
\hline Author & $\begin{array}{l}\text { Firesmith } \\
(2004)\end{array}$ & $\begin{array}{l}\text { Takabi } \\
\text { et al. } \\
(2010)\end{array}$ & $\begin{array}{l}\text { Brock and } \\
\text { Goscinski } \\
(2010)\end{array}$ & $\begin{array}{l}\text { Zissis and } \\
\text { Lekkas } \\
(2012)\end{array}$ & $\begin{array}{l}\text { Mapp } \\
\text { et al. } \\
(2014)\end{array}$ \\
\hline Confidentiality & $\sqrt{ }$ & $\sqrt{ }$ & $\sqrt{ }$ & $\sqrt{ }$ & $\sqrt{ }$ \\
\hline Integrity & $\sqrt{ }$ & $\sqrt{ }$ & $\sqrt{ }$ & $\sqrt{ }$ & $\sqrt{ }$ \\
\hline Availability & $\sqrt{ }$ & $\sqrt{ }$ & $\sqrt{ }$ & $\sqrt{ }$ & $\sqrt{ }$ \\
\hline Non-repudiation & $\sqrt{ }$ & & & & $\sqrt{ }$ \\
\hline Authenticity & & & $\sqrt{ }$ & $\sqrt{ }$ & $\sqrt{ }$ \\
\hline Reliability & & & $\sqrt{ }$ & $\sqrt{ }$ & \\
\hline
\end{tabular}

\subsection{Confidentiality}

One of the common security components is the protection of data by allowing only the intended recipient to read the information. This refers to confidentiality. Data should be handled correctly to prevent unauthorised exposure (Firesmith 2004). Brock and Goscinski (2010) has characterize security concerns of clouds by proposing a Cloud Security Framework (CSF) that takes into consideration cloud infrastructure protection to ensure confidentiality. Data can be protected by applying access controls, authentication and authorization while handling data effectively.

\subsection{Integrity}

The ability of a system or mechanism to detect changes or modifications to an original data ensures integrity. Some techniques implement integrity across a packet header and/or data field by creating a hash across the contents of the packet (Firesmith 2004). A systems integrity approach will review the architecture of a system and its implementation. A system designed and maintained with important aspects, which include contingency planning for power failures and disaster recovery, is also part of a system integrity (Firesmith 2004; Mapp et al. 2014). 


\subsection{Availability}

Availability is the idea that the data is accessible to all authorised users at all times. Its unavailability may occur in a physical way, as the failure of critical network components, power disruptions, and physical plant disruptions, either malicious or natural (Firesmith 2004; Takabi et al. 2010). Availability can also be impacted in a logical way, in the form of improper addressing or routing, and through the use of DenialOf-Service attacks, which are the deliberate insertion of unwanted data into the network (Brock and Goscinski 2010). This is often associated with address spoofing, which associates the introduction of unwanted data with a trusted end node. Zissis and Lekkas (2012) recommended user-specific security components for users.

\subsection{Non-repudiation}

The purpose of non-repudiation is to assign attribution, i.e. provenance, to a message that a third party could verify and be confident that it cannot be disputed. It can also prevent a recipient of a message from denying a message was received. Firesmith (2004) developed a detailed specification which attempts to provide a comprehensive security framework. It consists of nine layers: access control, attack harm detection, nonrepudiation, integrity, security auditing, physical protection, privacy and confidentiality, recovery, and prosecution. This framework provides a detailed analysis of the required functionality and therefore is able to serve as a reference model. This framework is widely adopted but has not been addressed within the cloud context. It was created at a general level to provide an overview of security components in information systems.

\subsection{Authenticity}

The authenticity of data refers to its original conception by its owner or author. Maintaining this relationship of data and network communications is performed with the use of public key encryption and a process called digital signing (Brock and Goscinski 2010; Zissis and Lekkas 2012). To create a digital signature, a hash is created across the data. A hash is sometimes referred to as a message that ensures the data is coming from an authentic source (Mapp et al. 2014). When ownership of a digital signature secret key is bound to a specific user, it demonstrates that the data was sent by a valid user. Thus, authenticating the source of data.

\subsection{Reliability}

Reliability refers to the ability of a system to provide consistent intended service (Brock and Goscinski 2010; Zissis and Lekkas 2012). Mapp et al. (2014) suggested a security framework using capabilities that are required to provide the operational reliability and flexibility needed in cloud environments. The proposed functions are developed into mechanisms using a capability-based approach. The development is implemented for an e-Health system that monitors patients. However, it is described as a process and does not have specific security components for cloud storage. 


\section{International and Industry Standards, Best Practice, and Guidelines}

The interest in cloud computing has led an explicit and constant effort to assess the latest trends in security (Honer 2013). The interest in cloud computing has led an explicit and constant effort to assess the latest trends in security (Honer 2013). Effective governance in cloud computing environments follows from well-developed information security processes as part of the organization's obligations (CSA 2013a; Srinivasan and Rodrigues 2012). In this section, IT industry standards in relation to promoting security are reviewed.

When the cloud was first introduced, the Cloud Security Alliance (CSA), a non-profit organization developed and published cloud security best practice (CSA 2009). Almost all major cloud providers (such as Amazon, Oracle, RedHat, and Salesforce) are members of the CSA. Their efforts include identifying the top concerns. A survey of industry experts was conducted by CSA to collect professional opinion of the vulnerabilities within cloud computing. In the latest edition, experts have identified data loss and breaches, and insecure APIs as the critical concerns to cloud security (CSA 2013a, 2013c). A compliance standard, called Cloud Control Matrix (CCM), was developed to provide standard security controls that can guide providers and help users in the assessment of the risks associated with a provider (CSA 2013b). The CCM is specifically designed as a control framework with security concepts aligned to CSA guidance in 13 domains. It also describes the relationship with industry-accepted security standards, regulations, and control frameworks (such as COBIT, ISO 27001/ 27002, PCI, NIST etc.) (CSA 2013b).

The National Institute of Standards and Technology, Security and Privacy Controls for Federal Information Systems and Organizations (NIST SP 800-53 Revision 4) was created to assist organizations in making the appropriate selection of security controls for information systems by introducing security control baselines (NIST 2013). Security control baselines are used as a starting point for the security control selection process and are based on the security category and associated impact level of information systems determined in accordance with FIPS Publication 199 and FIPS Publication 200 (NIST 2004). The baselines address the security needs of a comprehensive and varied set of constituencies, and are developed from several assumptions, including common environmental, operational, and functional considerations. However, the baselines also assume typical concerns facing common information systems (NIST 2013) but not specifically in the context of a cloud or cloud storage. Moreover, the suggestion of security protections based on categories of impact (low, moderate and high) has also not been included in the latest revision.

The European Network and Information Security Agency (ENISA) developed an authoritative security reference that listed risks, vulnerabilities, and a survey of related research recommendations. It consists of a report and practical guides designed for managing security in the cloud. In the asset management section, security measures highlight the point that service providers should review user data sensitivity. Another recommendation is that providers request information from users whether deploying their data in the cloud is deemed as sensitive enough to require additional security 
protection. Service providers are also encouraged to apply appropriate segregation between systems with different classifications (Catteddu and Hogben, 2009). The recommendation was only made in general and then only if there are sensitive data.

The United Kingdom Centre for the Protection of National Infrastructure (CPNI) has also provided critical security controls for cyber defence as baselines for high-priority information security measures and controls (CPNI 2014b). They can be applied across an organization to improve its cyber defence. The Council on Cybersecurity is coordinating the development of these controls. In their guidelines, the 20 controls (and sub-controls) concentrate on technical measures and activities. The main goal is assisting organizations in prioritising efforts to secure against the current and most common attacks. Besides that, comprehensive security should take into account other areas of security such as policy, organizational structure, and physical security. CPNI has added these in their latest guideline publication (CPNI 2014a). However, this guideline has not discussed cloud security in depth but some recommendations can also be applied to the cloud context.

In 2011, the Australian Signals Directorate (ASD) published 35 best practice strategies to mitigate targeted cyber intrusions (ASD 2014b) but it was simplified into four top mitigation strategies in 2012 (ASD 2012) focusing on application whitelisting, patching applications and operating systems, using the latest version, and minimising administrative privileges. The strategies are ranked in order of overall effectiveness and are based on ASD's analysis of reported security incidents and vulnerabilities. These are derived from ASD security testing and audits on Australian government networks. At the same time, the top four mitigation strategies are expected to effectively help in achieving a defence-in-depth ICT system. The combination of all four strategies, if correctly implemented, will protect an organization from low to moderately sophisticated intrusion attempts.

The Australian Government Information Security Manual (ISM) was published in 2014 and is the standard which governs the security of government ICT systems (ASD 2014a). It has 15 security aspects including: physical security, personnel security, communications security, information technology security, product security, media security, software security, email security, access control, secure administration, network security, cryptography, cross-domain security, data transfers and content filtering, and working off-site. There is an interesting section on protecting classified information and suggestions on encryption methods to protect confidential, secret and top secret information as shown in Table 1. The ISM comprises three documents targeting different levels within the organization, making the ISM accessible to more users and promoting information security awareness in Australian government agencies.

These industry-accepted standards, guidelines and best practice are reviewed and mapped against the security requirements discussed in the previous section (Brock and Goscinski 2010; Firesmith 2004; Mapp et al. 2014; Takabi et al. 2010; Zissis and Lekkas 2012). The Cloud Control Matrix (CCM) has been used as a reference document to crosscheck with other standards. NIST and ENISA are already included in CCM but are separated in this research in the context of cloud storage as it discusses information security in general. CPNI and ASD are not included in CCM as they provide the latest security guidelines and therefore have been thoroughly analysed. The summary is shown in Table 2. 
Table 2. Mapping of components to existing security controls in international and industry standards, guidelines, best practice

\begin{tabular}{l|l|l|l|l|l}
\hline Organization & $\begin{array}{l}\text { CSA } \\
(2013 \mathrm{~b})\end{array}$ & $\begin{array}{l}\text { NIST } \\
(2013)\end{array}$ & $\begin{array}{l}\text { ENISA } \\
(2009)\end{array}$ & $\begin{array}{l}\text { CPNI } \\
(2014 \mathrm{~b})\end{array}$ & $\begin{array}{l}\text { ASD } \\
(2014 \mathrm{a})\end{array}$ \\
\hline Confidentiality & $\sqrt{ }$ & $\sqrt{ }$ & $\sqrt{ }$ & $\sqrt{ }$ & $\sqrt{ }$ \\
\hline Integrity & $\sqrt{ }$ & $\sqrt{ }$ & $\sqrt{ }$ & $\sqrt{ }$ & $\sqrt{ }$ \\
\hline Availability & $\sqrt{ }$ & $\sqrt{ }$ & $\sqrt{ }$ & $\sqrt{ }$ & $\sqrt{ }$ \\
\hline Non-repudiation & $\sqrt{ }$ & & & & \\
\hline Authenticity & $\sqrt{ }$ & & $\sqrt{ }$ & $\sqrt{ }$ & $\sqrt{ }$ \\
\hline Reliability & & & $\sqrt{ }$ & $\sqrt{ }$ & $\sqrt{ }$ \\
\hline
\end{tabular}

\section{Protecting Sensitive Enterprise Data in Cloud Storage}

The Institute for Security and Open Methodologies (ISECOM) states that security provides protection where a separation is created between the assets and the threat (ISECOM 2001). These separations are generically called "controls" and sometimes include changes to the asset or the threat. The degree of resistance to or protection from harm may apply to any vulnerable and valuable asset, such as a person, community, nation, or enterprise organizations.

The cloud has enhanced security in many ways. For instance, the cloud provider is trusted to implement better and more recent security technologies and practices than the data owner (Ryan 2013). Conversely, data is stored outside the control of the data owner, which inevitably introduces security issues (Srinivasan and Rodrigues 2012; Subashini and Kavitha 2011; Suntharam et al. 2013). Many controls in the cloud, for example in securing access, are tackled by controls implemented by organizations and CSPs to secure access to sensitive data (Al-sabri and Al-saleem 2013; Calero et al. 2010; Kamara et al. 2011; Zhao and Yue 2014). Most CSPs are reluctant to decrease the efficiency of accessing the cloud storage as users expect similarly efficient access to secured data as with plain data. Some of the drawbacks of having excessive security controls in the cloud are (Honan 2012; Zarandioon et al. 2012; Zhang and Chen 2012; Zhao and Yue 2014; Zissis and Lekkas 2012):

- Decreased availability - the user may need to use a private cloud or an intranet to be able to access data in a controlled network. Some organizations require a security measure to be installed such as a Virtual Private Network.

- Needs robust infrastructure - a suitable infrastructure is needed to run encryption that is able to decrypt and encrypt data stored in the cloud. Misconfiguration may result in needing more computing resources.

- Costly data protection - some organizations require extra measures for identification, such as fingerprint readers and iris scanners. These are expensive and prone to error. The fingerprints are also a single piece of data that resembles a unique identity and can be stolen.

- Inconvenience - the most secure system has reduced use if it is hard to access. Requiring users to remember complicated passwords might keep data safer, but 
users may easily forget their passwords. This may require users to reset passwords and remember new combinations.

- Some data needs less protection - during the formative years of the web, as everything went online, passwords worked well. This was mainly due to the small amount of data to protect. Currently, having a single level of protection i.e. password may be insufficient as the threat landscape has evolved.

Security issues in the cloud have surrounded enterprise organizations with the growth of data held in cloud storage. As mentioned in the previous section, cloud storage has been widely utilized by users. Table 3 provides a brief representation of regional information on Internet users (adoption percentage) and the number of cloud storage users (based on the percentage of Internet users) for year 2019. The projection depicts the rise of cloud storage adoption rate by global users and enterprise organizations.

Table 3. Regional cloud storage users by 2019 (Cisco 2014)

\begin{tabular}{l|l|l}
\hline Region & $\begin{array}{l}\text { Internet users in millions } \\
(\% \text { of population })\end{array}$ & $\begin{array}{l}\text { Cloud storage users in millions } \\
\text { (\% of internet users) }\end{array}$ \\
\hline Asia Pacific & $2,022(49 \%)$ & $1,176(58 \%)$ \\
\hline Central and Eastern Europe & $321(66 \%)$ & $134(42 \%)$ \\
\hline Latin America & $355(54 \%)$ & $141(40 \%)$ \\
\hline Middle East and Africa & $401(25 \%)$ & $65(16 \%)$ \\
\hline North America & $311(83 \%)$ & $257(83 \%)$ \\
\hline Western Europe & $341(80 \%)$ & $272(80 \%)$ \\
\hline
\end{tabular}

The concerns towards the security of data in the cloud have encouraged studies undertaken in the area of cloud storage security; this involved developing frameworks to guide enterprise users and CSPs. This has encouraged governing bodies and agencies to publish standards, best practice and guidelines that can be used as references by enterprise organizations adopting cloud storage into their global cloud solutions. Cloud Security Alliance (CSA) in particular has been actively developing guidelines and the Cloud Control Matrix (CCM) is among the important ones that map the controls to other standards protection domains. The National Institute of Standards and Technology (NIST) published NIST 800-53 R4 that presented a risk-based approach in establishing responsibilities for implementing important controls in the cloud. Existing standards, best practices, and guidelines has provide recommendations for the cloud in general but has not put specific emphasis on cloud storage.

Previous research has shown that organizations and CSPs have implemented many controls to ensure security and data protection. However, some measures involve many controls that most enterprise are reluctant to impose, as it is likely to decrease the efficiency of accessing cloud storage. Applying controls based on goal-driven security components and concerns is proposed to protect data efficiently in cloud storage. The proposed solution can be used to better assess an enterprise security goal and policy implementation whether existing policies and controls are in place, measures have been 
taken or vice versa. The next section discusses methods for deriving security metrics from security goals.

\section{Methods for Deriving Security Metrics from Security Goals}

According to (Kassou and Kjiri 2012), there are three approaches that support metrics derivation from goals: GQM (Goal-Question-Metric) approach (Basili et al. 1994), GAM (Goal-Argument-Metric) (Cyra and Górski 2008) and BSC (Balanced Scorecard Framework) (Buglione and Abran 2000). GQM Approach provides an outline of process that defines goals, refining them into questions and then specifying measurements and finally data to be collected. GAM is a goal-oriented methodology for defining measurement plans. BSC is a framework that look into several dimensions for describing, implementing and managing strategy at different levels of an organization by linking objectives, initiatives and measures to an organization's strategy.

Considering the purpose and the general approach (top down derivation and bottom-up interpretation) GQM and GAM look the same. The differences relate to the way of defining and maintaining the relationship between the measurement goals and the metrics. In GAM, the goals and sub-goals are denoted as claims and then the analysis focuses on classifying which data and which properties of the data (further sub-goals) are needed to fulfill these claims whereas in GQM referring to a goal, several questions are defined in such a way that obtaining the answers to the questions leads to the achievement of the measurement goal then based on the questions, metrics are defined, which provide quantitative information then treated as answers to the questions (Basili et al. 1994).

GQM Goals are referred to as a mission, while BSC goals are referred to a certain perspective and a certain particular tier in the organizational pyramid (hierarchy). Besides, GQM can be defined as a technique for deriving quantitative measures from a list of goals while BSC can be viewed as performance management framework that uses a GQM-like technique to derive the indicators (Buglione and Abran 2000).

Table 4 shows GQM, GAM and BSC comparison and similarities.

Table 4. GQM, GAM and BSC approach (adapted from (Buglione and Abran 2000; Kassou and Kjiri 2012))

\begin{tabular}{l|l|l|l}
\hline \multirow{2}{*}{ Approach level } & \multicolumn{3}{|l}{ Measurement approach } \\
\cline { 2 - 4 } & GQM & GAM & BSC \\
\hline $\begin{array}{l}\text { Conceptual } \\
\text { - Objects }\end{array}$ & Goal & Claim & Goal \\
\hline $\begin{array}{l}\text { Operational } \\
\text { - Assessment }\end{array}$ & Question & Assertion & Driver \\
\hline $\begin{array}{l}\text { Quantitative } \\
\text { - Objective/Subjective }\end{array}$ & Metric & Metric & Indicator \\
\hline
\end{tabular}




\section{Goal-Question-Metric (GQM) Approach}

This section will present the Goal-Question-Metrics and provide example of its application in security research. The Goal-Question-Metric (GQM) approach is based upon the assumption that for an organization to measure in a purposeful way it must first specify goals for itself and its projects, then it must match those goals to the data that are intended to define those goals operationally, and finally provide a framework for interpreting the data with respect to the stated goals. The GQM paradigm (Basili et al. 1994; Basili 1992, 1993) is based on the theory that all measurement should be goal-oriented i.e., there has to be some rationale and need for collecting measurements $\&$ each measurement collected is stated in terms of the major goals. Questions are then derived from the goals and help to refine, articulate, and determine if the goals can be achieved. The metrics or measurements that are collected are then used to answer the questions in a quantifiable manner.

A GQM Model is a hierarchical structure as presented in Fig. 3 starting from a goal (specifying purpose of measurement, object/issue to measured, and viewpoint from which measure is taken). GQM defines a measurement model on three levels:

1. Conceptual level (Goal)

A goal is defined for an object, for a range of reasons, with respect to different models of value, from different perspectives and relative to a specific domain.

2. Operational level (Question)

A set of questions is utilized to define models of the object of study and after that emphases on that object to describe the evaluation or accomplishment of a particular goal.

3. Quantitative level (Metric)

A set of measurements, taking into account the models, associated with every question in order to answer it quantifiably.

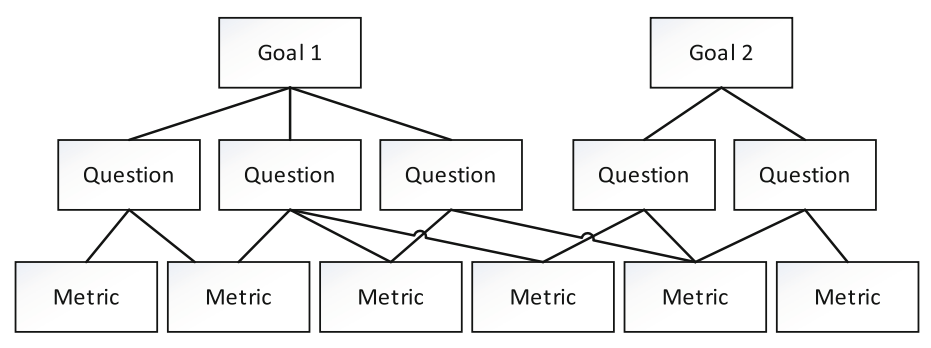

Conceptual Level

Operational Level

Quantitative Level

Fig. 3. GQM hierarchical approach

\section{Application of GQM}

Security requirements are actually a set of conditions that describe properties such as confidentiality, integrity, availability, authenticity, and non-repudiation etc. of the systems security goal (Islam and Falcarin 2011). It is a set of requirements which consider 
organization policies, security goals and security policies. The security requirements identified here are based on the security goal identified in cloud storage security challenges and threats in previous research (Yahya et al. 2014, 2015). According to (Kassou and Kjiri 2012), there are four steps to build SM using GQM as follows.

\section{Building Security Viewpoint}

For each security goal, we need to define the viewpoint of the security context related to control that is provided by other viewpoints: Infrastructure, Governance etc.

1. Developing Goals

Based on Cloud Security Alliance Control Matrix (CCM) and other controls from the literature, we have identified security goals; confidentiality, integrity, availability, non-repudiation, authenticity, and reliability (Yahya et al. 2014, 2015). Each security goals are have been related to the security of data in cloud storage.

2. Refining Security Goals into questions and deriving metrics

We present in Table 5, an example of security goal; showing the confidentiality of data stored in cloud storage can be achieved by having controls such as identity management, access management and controls.

Table 5. Example of GQM application (In assessing the confidentiality of data accessed in cloud storage)

\begin{tabular}{|c|c|c|c|}
\hline \multicolumn{2}{|l|}{ Goal } & \multirow{2}{*}{$\begin{array}{l}\text { Question } \\
\text { Co1. Identity Management Sub- } \\
\text { questions: }\end{array}$} & \multirow[t]{2}{*}{ Metric } \\
\hline Purpose & Assessing & & \\
\hline Issue/Component & the Confidentiality & $\begin{array}{l}\text { Co1.1. Implementation of identity } \\
\text { management policy? }\end{array}$ & Rating score* \\
\hline Object/Process & $\begin{array}{l}\text { of Data accessed in } \\
\text { cloud storage }\end{array}$ & $\begin{array}{l}\text { Co1.2. Has user-based } \\
\text { authentication process/solutions? }\end{array}$ & Yes/No \\
\hline Where & in organization & $\begin{array}{l}\text { Co1.3. Uses standards to delegate } \\
\text { authentication? }\end{array}$ & Rating score* \\
\hline \multirow[t]{7}{*}{ Viewpoint } & $\begin{array}{l}\text { from the } \\
\text { stakeholder's } \\
\text { viewpoint }\end{array}$ & Co2. Access management & \\
\hline & & $\begin{array}{l}\text { Co2.1. Implementation of access } \\
\text { management policy? }\end{array}$ & Rating score* \\
\hline & & $\begin{array}{l}\text { Co } 2.2 \text {. How many access points to } \\
\text { the cloud storage? }\end{array}$ & $\begin{array}{l}\text { Number of } \\
\text { access points }\end{array}$ \\
\hline & & $\begin{array}{l}\text { Co2.3. Percentage of enforcement } \\
\text { points? }\end{array}$ & Percentage \\
\hline & & . & \\
\hline & &.. & \\
\hline & & $\ldots$ & \\
\hline
\end{tabular}

* Rating Score 1 - No Plan to Implement, 2 - Planning to Implement, 3 - Do not know/Not sure, 4

- Partially Implement, 5 - Fully Implement 


\section{Detailing metrics}

A set of questions and sub questions follows the key indicator. An example includes, identity management, authentication, access management, access points etc.

The four steps is explained in the context of the stakeholders. In this scenario, the stakeholders are IT managers in data centers that manages cloud storage. IT managers are responsible to maintain an organization's effectiveness and efficiency by defining, delivering, and supporting strategic plans for implementing secure information technologies i.e. ensuring that security policy and controls are in place and implemented in an organization. Therefore, security goals in an organization can be assessed and achieved.

\section{Conclusion}

We have presented a Goal-Question-Metric (GQM) approach to define Security Metrics (SM) to assess the security control features of cloud storage systems. Once defined, these SM can be applied to evaluate the level of security through the following assessment process.

1. Characterize environment assessment - evaluate cloud storage (infrastructure, storage, etc.)

2. Identify Measurement Goals - select security goals and their associated metrics

3. Develop Measurement Plan - assess security controls based on existing studies (frameworks and standards, etc.)

4. Use Measurement Results - use assessment results for showing compliance to cloud storage security framework

Evaluating cloud storage security can be useful for organizations that use cloud storage to: (i) Use the assessment result to ensure the safety of their cloud storage; (ii) Enhance their security implementations to be aligned with their current or target security policy (iii) Improve the quality of their security processes according to the security capability level they are targeting.

In this paper we have presented several approaches for deriving security metrics from security goals; GQM, GAM \& BSC. We have also made a comparison of the approach in the context of security. GQM was introduced as a solution to present our work. A brief explanation on how we develop the security metrics are described with details on the process. Our solution proposes a GQM approach to derive security metrics from cloud storage security key indicators (focusing on control characteristics) and their related security goals. Metrics that we have defined and their implementation evidence can used to support organizations in assessing security in cloud storage especially for control features, to enhance the protections in cloud storage.

\section{References}

Al-sabri, H.M., Al-saleem, S.M.: Building a cloud storage encryption (CSE) architecture for enhancing cloud security. IJCSI Int. J. Comput. Sci. 10(2), 259-266 (2013) 
ASD: Top four mitigation strategies to protect your ICT system. Australian Signals Directorate (ASD) (2012). http://www.asd.gov.au/publications/protect/Top_4_Mitigations.pdf. Accessed 22 Aug 2014

ASD: Australian Government Information Security Manual Controls. Australian Signals Directorate (ASD), Australian Signals Directorate (2014a). http://www.asd.gov.au/publications/ Information_Security_Manual_2014_Principles.pdf. Accessed 12 Oct 2014

ASD: Strategies to mitigate targeted cyber intrusions - mitigation details. Australian Signals Directorate (ASD). Australian Signals Directorate (2014b). www.asd.gov.au/publications/ Mitigation_Strategies_2011.pdf. Accessed 16 Aug 2014

Baldwin, D.: The concept of security. Rev. Int. Stud. 23(01), 5-26 (1997)

Basili, V.R.: Software Modeling And Measurement: The Goal/Question/Metric Paradigm. Quality (1992)

Basili, V.R.: Applying the Goal/Question/Metric Paradigm in the Experience Factory. Software Quality Assurance and Measurement A Worldwide Perspective (1993)

Basili, V.R., Caldiera, G., Rombach, H.D.: Goal Question Metric Paradigm. Encyclopedia of Software Engineering. Wiley, New York (1994). doi:10.1002/0471028959.sof142

Brock, M., Goscinski, A.: Toward a framework for cloud security. In: Hsu, C.H., Yang, L.T., Park, J.H., Yeo, S.S. (eds.) Algorithms and Architectures for Parallel Processing. ICA3PP 2010. LNCS, vol. 6082, pp. 254-263. Springer, Heidelberg (2010)

Buglione, L., Abran, A.: Balanced scorecards and GQM: what are the differences? In: 3rd European Software Measurement Conference, FESMA-AEMES 2000, pp. 18-20 (2000)

Calero, J.M.A., Edwards, N., Kirschnik, J., Wilcock, L., Wray, M.: Toward a multi-tenancy authorization system for cloud services. Secur. Priv. IEEE 8(6), 48-55 (2010)

Catteddu, D., Hogben, G.: Cloud computing: benefits, risks and recommendations for information security. White Paper. European Network and Information Security Agency (ENISA) (2009)

Chang, V., Ramachandran, M.: Towards achieving data security with the cloud computing adoption framework. IEEE Trans. Serv. Comput. 9(1), 138-151 (2016). doi:10.1109/TSC.2015.2491281

Cisco: Cisco Global Cloud Index : Forecast and Methodology, 2011-2016. White Paper, 1-41 (2014). http://www.cisco.com/en/US/solutions/collateral/ns341/ns525/ns537/ns705/ns1175/ Cloud_Index_White_Paper.html\#wp9000816

Colobran, M.: Modeling human perceived security: a conceptual framework and its application to health. Comput. Hum. Behav. 62, 1-8 (2016). doi:10.1016/j.chb.2016.03.050

CPNI: Reducing the cyber risk in 10 critical areas. White Paper, Centre for the Protection of National Infrastructure (CPNI) (2014a). https://www.gov.uk/government/uploads/system/ uploads/attachment_data/file/395716/10_steps_ten_critical_areas.pdf. Accessed 22 Aug 2014

CPNI: The critical security controls for effective cyber defense V5.0 report. Centre for the Protection of National Infrastructure (CPNI) (2014b). http://www.cpni.gov.uk/documents/publications/ 2014/2014-04-11-critical-security-controls.pdf?epslanguage=en-gb. Accessed 22 Aug 2014

CSA: Security Guidance for Critical Areas of Focus in Cloud Computing V2.1, White Paper. Cloud Security Alliance (CSA) (2009). doi:10.1016/S1353-4858(99)90042-9

CSA: Top Threats to Cloud Computing V1.0 Report. Cloud Security Alliance (CSA) (2010). https://cloudsecurityalliance.org/topthreats/csathreats.v1.0.pdf. Accessed 22 Aug 2014

CSA: Cloud computing vulnerability incidents : a statistical overview report. Cloud Security Alliance (CSA) (2013a). https://cloudsecurityalliance.org/download/cloud-computingvulnerability-incidents-a-statistical-overview/. Accessed 22 Aug 2014

CSA: The Cloud Control Matrix V3.0.1. White Paper, Cloud Security Alliance (CSA) (2013b). https://cloudsecurityalliance.org/download/cloud-controls-matrix-v3-0-1. Accessed 22 Aug 2014

CSA: The Notorious Nine: Cloud Computing Top Threats in 2013 Report. Cloud Security Alliance (CSA) (2013c). https://downloads.cloudsecurityalliance.org/initiatives/top_threats/ The_Notorious_Nine_Cloud_Computing_Top_Threats_in_2013.pdf. Accessed 22 Aug 2014 
Cyra, L., Górski, J.: Extending GQM by arguement structures. In: CEE-SET 2007, vol. 44(5), pp. 26-39 (2008). doi:10.1016/j.ipm.2008.03.002

Firesmith, D.: Specifying reusable security requirements. J. Object Technol. 3(1), 61-75 (2004). doi:10.5381/jot.2004.3.1.c6

Gonzales, D., Kaplan, J., Saltzman, E., Winkelman, Z., Woods, D.: Cloud-trust - a security assessment model for infrastructure as a service (IaaS) clouds. IEEE Trans. Cloud Comput. 14 (2015). doi:10.1109/TCC.2015.2415794

Granneman, J.: IT security frameworks and standards: Choosing the right one (2013). http:// searchsecurity.techtarget.com/tip/IT-security-frameworks-and-standards-Choosing-the-rightone. Accessed 12 Aug 2015

GTISC and GTRI (2013): Emerging cyber threats report 2014. Georgia Tech Information Security Center (GTISC) and Georgia Tech Research Institute (GTRI), Georgia Tech Cyber Security Summit (2013). https://www.gtisc.gatech.edu/pdf/Threats_Report_2014.pdf. Accessed 22 Aug 2014

Honan, M.: Kill the password: why a string of characters can't protect us anymore. WIRED, pp. 9-16 (2012)

Honer, P.: Cloud computing security requirements and solutions: a systematic literature review. In: 19th Twente Student Conference on IT, Enshede, The Netherlands (2013). doi:10.1007/ 978-3-642-40861-8_42

Hubbard, D.W.: How to Measure Anything: Finding the Value of Intangibles in Business. Wiley, New York (2014)

ISECOM: ISECOM - Open Source Security Testing Methodology Manual (OSSTMM). Institute for Security and Open Methodologies (ISECOM) (2001). http://www.isecom.org/mirror/ OSSTMM.3.pdf. Accessed 22 Aug 2014

Islam, S., Falcarin, P.: Measuring security requirements for software security. In: IEEE 10th International Conference in Cybernetic Intelligent System (CIS), pp. 70-75 (2011). doi:10.1109/CIS.2011.6169137

Jahedi, S., Méndez, F.: On the advantages and disadvantages of subjective measures. J. Econ. Behav. Organ. 98, 97-114 (2014). doi:10.1016/j.jebo.2013.12.016

Jones, M.T.: Anatomy of a cloud storage infrastructure: models, features, and internals. White Paper. Developer Works, IBM Corporation 2010. http://www.ibm.com/developerworks/ cloud/library/cl-cloudstorage/cl-cloudstorage-pdf.pdf. Accessed 8 Sept 2014

Ju, J., Wu, J., Fu, J., Lin, Z.: A survey on cloud storage. J. Comput. 6(8), 1764-1771 (2011). doi:10.4304/jcp.6.8.1764-1771

Kamara, S., Papamanthou, C., Roeder, T.: CS2 : a searchable cryptographic cloud storage system. Microsoft Research, pp. 1-25 (2011)

Kassou, M., Kjiri, L.: A goal question metric approach for evaluating security in a service oriented architecture context. Int. J. Comput. Sci. Issues 9, 1-12 (2012)

Mapp, G., Aiash, M., Ondiege, B., Clarke, M.: Exploring a new security framework for cloud storage using capabilities. In: Proceedings of IEEE 8th International Symposium on Service Oriented System Engineering (SOSE), pp. 484-489 (2014). doi:10.1109/SOSE.2014.69

Mather, T., Kumaraswamy, S., Latif, S.: Cloud Security and Privacy: An Enterprise Perspective on Risks and Compliance. International Journal of Policy and Administration. O'Reilly Media Inc., Sebastopol (2009)

Microsoft: Security Threats. Microsoft Developer Network (MSDN) (2015). https://msdn.microsoft. com/en-us/library/cc723507.aspx. Accessed 22 Apr 2015

NIST: Standards for Security Categorization of Federal Information and Information Systems. National Institute of Standards and Technology (NIST), Special Publication FIPS 199 (2004) NIST: Security and Privacy Controls for Federal Information Systems and Organizations. National Institute of Standards and Technology (NIST), Special Publication 800-53 Revision 4 (2013) 
Payne, S.C.: A Guide to Security Metrics. SANS Institute (2006)

Pendleton, M., Garcia-Lebron, R., Xu, S.: A Survey on Security Metrics (2016). arXiv Preprint arXiv:1601.05792

Rouse, M.: What is storage? TechTarget (2005). http://searchstorage.techtarget.com/definition/ storage. Accessed 12 Mar 2015

Ryan, M.D.: Cloud computing security: the scientific challenge, and a survey of solutions. J. Syst. Softw. 86(9), 2263-2268 (2013). doi:10.1016/j.jss.2012.12.025

Sabahi, F.: Cloud computing security threats and responses. In: 2011 IEEE 3rd International Conference on Communication Software and Networks, pp. 245-249 (2011). doi:10.1109/ ICCSN.2011.6014715

Shaikh, F.B., Haider, S.: Security threats in cloud computing. In: 6th International Conference on Internet Technology and Secured Transactions, Abu Dhabi, UAE, 11-14 December 2011

Singh, R., Kumar, S., Agrahari, S.K.: Ensuring data storage security in cloud computing. IOSR J. Eng. 2(12), 17-21 (2012)

Srinivasan, M.K., Rodrigues, P.: State-of-the-art cloud computing security taxonomies - a classification of security challenges in the present cloud. In: ICACCI 2012, pp. 470-476 (2012)

Subashini, S., Kavitha, V.: A survey on security issues in service delivery models of cloud computing. J. Netw. Comput. Appl. 34(1), 1-11 (2011). doi:10.1016/j.jnca.2010.07.006

Suntharam, V.S., Reddy, K.V., Puspalatha, N.: Data storage security in cloud computing and verification of metadata by encryption. Int. J. Comput. Sci. Electron. Eng. 2(3), 1-9 (2013)

Takabi, H., Joshi, J.B.D., Ahn, G.J.: SecureCloud: towards a comprehensive security framework for cloud computing environments. In: Proceedings of International Computer Software and Applications Conference, pp. 393-398 (2010). doi:10.1109/COMPSACW.2010.74

Torkhani, F., Wang, K., Chassery, J.M.: Perceptual quality assessment of 3D dynamic meshes: subjective and objective studies. Signal Process. Image Commun. 31, 185-204 (2015). doi:10.1016/j.image.2014.12.008

Wang, C., Wang, Q., Ren, K., Cao, N., Lou, W.: Toward secure and dependable storage services in cloud computing. IEEE Trans. Serv. Comput. 5, 220-232 (2012). doi:10.1109/TSC.2011.24

Wu, J., Ping, L., Ge, X., Ya, W., Fu, J.: Cloud storage as the infrastructure of cloud computing. In: Proceedings of 2010 International Conference on Intelligent Computing and Cognitive Informatics, ICICCI 2010, pp. 380-383 (2010). doi:10.1109/ICICCI.2010.119

Yahya, F., Chang, V., Walters, R.J., Wills, G.B.: Security challenges in cloud storage. In: 6th IEEE International Conference on Cloud Computing Technology and Science. Enterprise Security 2014, pp. 1051-1056 (2014). doi:10.1109/CloudCom.2014.171

Yahya, F., Walters, R.J., Wills, G.B.: Modelling threats with security requirements in cloud storage. Int. J. Inf. Secur. Res. (IJISR) 5(2), 551-558 (2015)

Zarandioon, S., Yao, D(., Ganapathy, V.: K2C: cryptographic cloud storage with lazy revocation and anonymous access. In: Rajarajan, M., Piper, F., Wang, H., Kesidis, G. (eds.) SecureComm 2011. LNICSSITE, vol. 96, pp. 59-76. Springer, Heidelberg (2012). doi:10.1007/978-3-64231909-9_4

Zhang, R., Chen, P.: A dynamic cryptographic access control scheme in cloud storage services. In: Proceedings of 2012 8th International Conference on Computing and Networking Technology (INC, ICCIS and ICMIC), ICCNT 2012, pp. 50-55 (2012). doi:10.4156/ijipm. vol4.issue1.13

Zhao, R., Yue, C.: Toward a secure and usable cloud-based password manager for web browsers. Comput. Secur. 46, 32-47 (2014). doi:10.1016/j.cose.2014.07.003

Zissis, D., Lekkas, D.: Addressing cloud computing security issues. Future Gener. Comput. Syst. 28(3), 583-592 (2012). doi:10.1016/j.future.2010.12.006 